Antony Unwin

Martin Theus

Heike Hofmann

\title{
Graphics of \\ Large Datasets
}

Visualizing a Million

Springer 


\section{Contents}

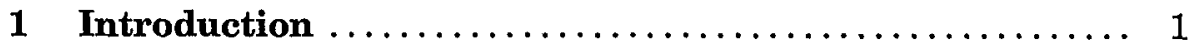

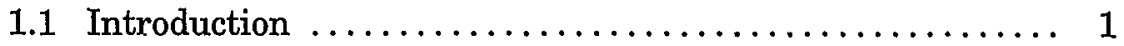

1.2 Data Visualization........................... 4

1.3 Research Literature ......................... 7

1.4 How Large Is a Large Dataset? .................. 9

1.5 The Effects of Largeness . . . . . . . . . . . . . . . . . 17

1.5.1 Storage ................................ 18

1.5.2 Quality .............................. 19

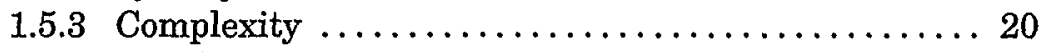

1.5.4 Speed .............................. 20

1.5.5 Analyses.............................. 21

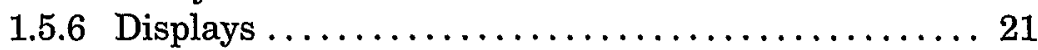

1.5.7 Graphical Formats ..................... 22

1.6 What Is in This Book $\ldots \ldots \ldots \ldots \ldots \ldots \ldots \ldots \ldots \ldots . \ldots \ldots, 22$

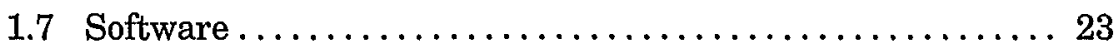

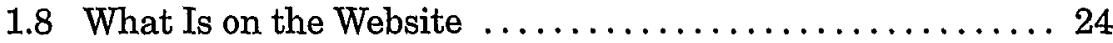

1.8.1 Files and Code for Figures................. 24

1.8.2 Links to Software....................... 24

1.8.3 Datasets............................. 25

1.9 Contributing Authors $\ldots \ldots \ldots \ldots \ldots \ldots \ldots \ldots \ldots \ldots, 26$

\section{Part I Basics}

2 Statistical Graphics $\ldots \ldots \ldots \ldots \ldots \ldots \ldots \ldots \ldots \ldots \ldots \ldots \ldots$

2.1 Introduction $\ldots \ldots \ldots \ldots \ldots \ldots \ldots \ldots \ldots \ldots \ldots \ldots \ldots, \ldots \ldots \ldots$

2.2 Plots for Categorical Data ...................... 31

2.2.1 Barcharts and Spineplots for Univariate Categorical Data ....................... 32

2.2.2 Mosaic Plots for Multi-dimensional Categorical Data 33

2.3 Plots for Continuous Data ..................... 36 
2.3.1 Dotplots, Boxplots, and Histograms .......... 36

2.3.2 Scatterplots, Parallel Coordinates, and the Grand

Tour............................... 39

2.4 Data on Mixed Scales ....................... 44

2.5 Maps ................................. 47

2.6 Contour Plots and Image Maps $\ldots \ldots \ldots \ldots \ldots \ldots \ldots \ldots . \ldots 9$

2.7 Time Series Plots . . . . . . . . . . . . . . . . . . . . . 50

2.8 Structure Plots ........................... 51

$3 \quad$ Scaling Up Graphics $\ldots \ldots \ldots \ldots \ldots \ldots \ldots \ldots \ldots \ldots \ldots \ldots \ldots$

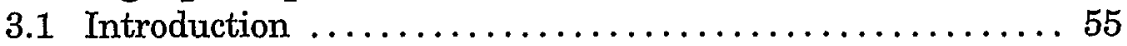

3.2 Upscaling as a General Problem in Statistics.......... 55

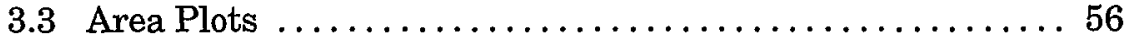

3.3.1 Histograms ....................... 57

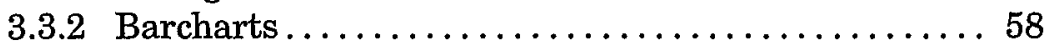

3.3.3 Mosaic Plots ......................... 60

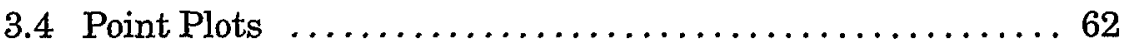

3.4.1 Boxplots ..........................62 62

3.4.2 Scatterplots ............................63 63

3.4.3 Parallel Coordinates .....................6. 65

3.5 From Areas to Points and Back ................. 67

3.5.1 $\alpha$-Blending and Tonal Highlighting ........... 69

3.6 Modifying Plots $\ldots \ldots \ldots \ldots \ldots \ldots \ldots \ldots \ldots \ldots \ldots \ldots \ldots \ldots \ldots$

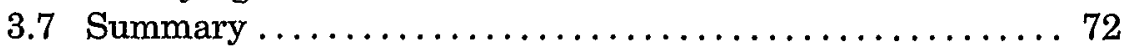

4 Interacting with Graphics $\ldots \ldots \ldots \ldots \ldots \ldots \ldots \ldots \ldots \ldots \ldots$

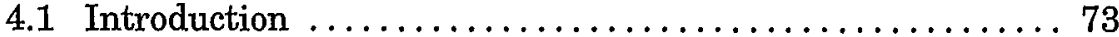

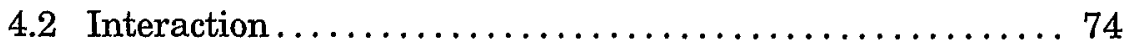

4.3 Interaction and Data Displays ................. 75

4.3 .1 Querying .............................. 75

4.3.2 Selection and Linking..................... 77

4.3.3 Selection Sequences ....................... 78

4.3.4 Varying Plot Characteristics .................. 82

4.3.5 Interfaces and Interaction .................. 84

4.3.6 Degrees of Linking . ...................... 86

4.3.7 Warnings and Redmarking .................. 87

4.4 Interaction and Large Datasets $\ldots \ldots \ldots \ldots \ldots \ldots \ldots \ldots$

4.4.1 Querying ............................. 88

4.4.2 Selection, Linking, and Highlighting . ......... 89

4.4.3 Varying Plot Characteristics for Large Datasets ..... 92

4.5 New Interactive Tasks $\ldots \ldots \ldots \ldots \ldots \ldots \ldots \ldots \ldots . \ldots 9$

4.5.1 Subsetting ............................ 98

4.5.2 Aggregation and Recoding.................. 99

4.5.3 Transformations $\ldots \ldots \ldots \ldots \ldots \ldots \ldots \ldots \ldots, \ldots 9$

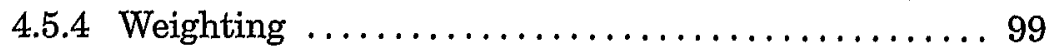


4.5.5 Managing Screen Layout.................... 101

4.6 Summary and Future Directions ................. 101

\section{Part II Applications}

5 Multivariate Categorical Data - Mosaic Plots ..........105

5.1 Introduction ............................... 105

5.2 Area-based Displays ........................ 105

5.2.1 Weighted Displays and Weights in Datasets .......107

5.3 Displays and Techniques in One Dimension ........... 107

5.3.1 Sorting and Reordering ...................110

5.3.2 Grouping, Averaging, and Zooming.............111

5.4 Mosaic Plots .................................... 113

5.4.1 Combinatorics of Mosaic Plots ................ 114

5.4.2 Cases per Pixel and Pixels per Case .............116

5.4.3 Calibrating the Eye...................... 116

5.4.4 Gray-shading ............................... 119

5.4.5 Rescaling Binsizes........................ 122

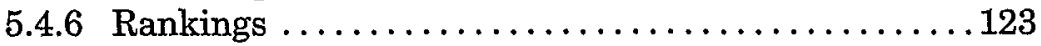

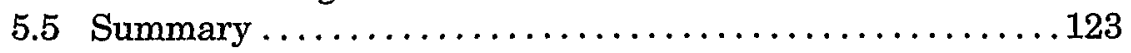

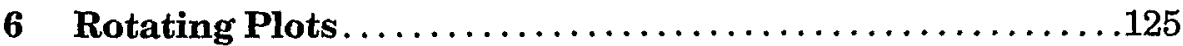

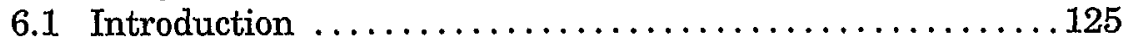

6.1.1 Type of Data .......................... 126

6.1.2 Visual Methods for Continuous Variables ..........127

6.1.3 Scaling Up Multiple Views for Larger Datasets...... 128

6.2 Beginning to Work with a Million Cases ............. 128

6.2.1 What Happens in GGobi, a Real-time System? . . . . . 128

6.2.2 Reducing the Number of Cases . . . . . . . . . . . . 129

6.2.3 Density Estimation .......................... 131

6.2.4 Screen Real Estate Indexing................... 134

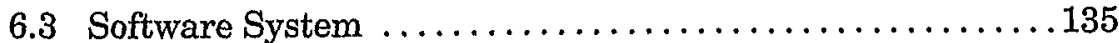

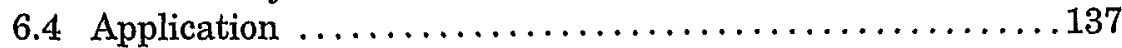

6.4.1 Data Description ........................ 137

6.4.2 Viewing a Tour of the Data ................. 137

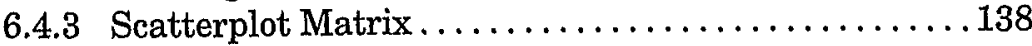

6.5 Current and Future Developments ................. 140

6.5.1 Improving the Methods ..................... 140

6.5.2 Software............................... 141

6.5.3 How Might These Tools Be Used? .............. 141 
7 Multivariate Continuous Data - Parallel Coordinates ....143

7.1 Introduction .............................143

7.2 Interpolations and Inner Products ................ 144

7.3 Generalized Parallel Coordinate Geometry............145

7.4 A New Family of Smooth Plots . . . . . . . . . . . . . . 149

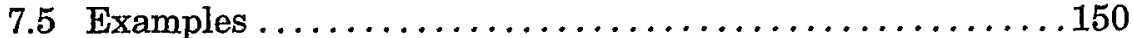

7.5.1 Automobile Data ........................150

7.5.2 Hyperspectral Data: Dealing with Massive Datasets . 152

7.6 Detecting Second-Order Structures ................154

7.7 Summary ............................... 155

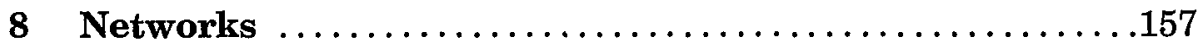

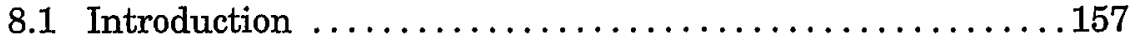

8.2 Layout Algorithms......................... 158

8.2.1 Simple Tree Layout......................... 159

8.2.2 Force Layout Methods . ....................161

8.2.3 Individual Node Movement Algorithms ..........162

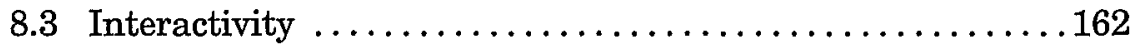

8.3.1 Speed Considerations ........................ 164

8.3.2 Interaction and Layout $\ldots \ldots \ldots \ldots \ldots \ldots \ldots \ldots \ldots$

8.4 NicheWorks ................................ 166

8.5 Example: International Calling Fraud . . . . . . . . . . . . 167

8.6 Languages for Description and Layouts............... 172

8.6.1 Defining a Graph ............................. 172

8.6.2 Graph Specification via VizML ...............173

8.7 Summary ................................. 174

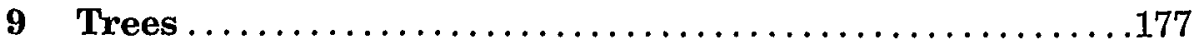

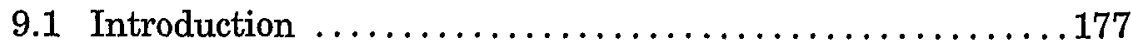

9.2 Growing Trees for Large Datasets.................. 178

9.2.1 Scalability of the CART Growing Algorithm ........179

9.2.2 Scalability of Pruning Methods ................ 181

9.2.3 Statistical Tests and Large Datasets ............. 183

9.2.4 Using Trees for Large Datasets in Practice ......... 184

9.3 Visualization of Large Trees . . . . . . . . . . . . . . . . . . 187

9.3.1 Hierarchical Plots .......................... 187

9.3.2 Sectioned Scatterplots ....................... 192

9.3.3 Recursive Plots ............................ 195

9.4 Forests for Large Datasets . . . . . . . . . . . . . . . . . . . . . . 198

9.5 Summary ................................202 


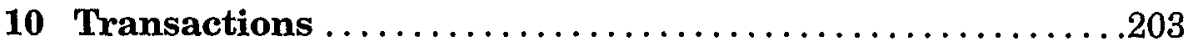

10.1 Introduction and Background...................203

10.2 Mice and Elephant Plots and Random Sampling........205

10.3 Biased Sampling ............................210

10.3.1 Windowed Biased Sampling ................211

10.3.2 Box-Cox Biased Sampling.................213

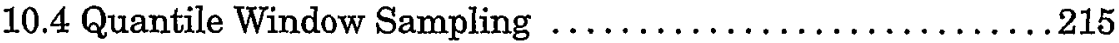

10.5 Commonality of Flow Rates $\ldots \ldots \ldots \ldots \ldots \ldots \ldots \ldots .221$

11 Graphics of a Large Dataset $\ldots \ldots \ldots \ldots \ldots \ldots \ldots \ldots \ldots \ldots \ldots \ldots$

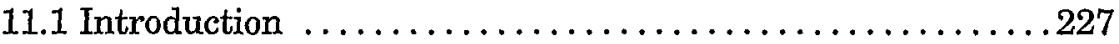

11.2 QuickStart Guide

Data Visualization for Large Datasets ................. . 228

11.3 Visualizing the InfoVis 2005 Contest Dataset .........229

11.3.1 Preliminaries ..........................229

11.3.2 Variables ............................230

11.3.3 First Analyses $\ldots \ldots \ldots \ldots \ldots \ldots \ldots \ldots \ldots \ldots \ldots . .230$

11.3.4 Multivariate Displays....................235

11.3.5 Grouping and Selection .....................239

11.3.6 Special Features.........................242

11.3.7 Presenting Results . .....................247

11.3.8 Summary .............................249

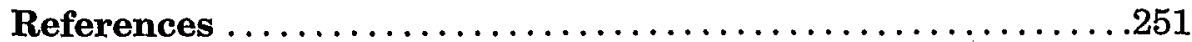

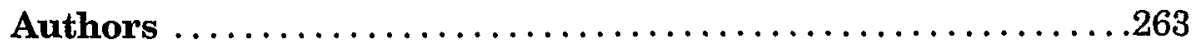

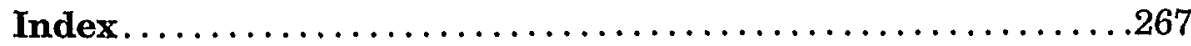

